SELECCIONES MATEMÁTICAS
Universidad Nacional de Trujillo
ISSN: $2411-1783$ (Online)
Vol. $05(02): 164-174(2018)$

\title{
Decaimiento Polinomial y Modelaje Numérico Computacional de la viga de Timoshenko con disipación parcial
}

\section{Polynomial Decay and Computational Numerical Modeling of Timoshenko beam with partial dissipation}

\author{
Frank Henry Acasiete Quispe ${ }^{*}$ and Neisser Pino Romero ${ }^{\dagger}$ \\ Received, Set. 27, 2018 \\ Accepted, Dec. 07, 2018
}

DOI: http://dx.doi.org/10.17268/sel.mat.2018.02.04

\begin{abstract}
Resumen
Estudiamos la estabilización uniforme de una clase de sistemas Timoshenko con disipación parcial de la viga. Nuestro resultado principal es demostrar que el semigrupo asociado a este modelo tiene decaimiento polinomial. Demostramos que el semigrupo decae polinomialmente a cero. El sistema decae polinomialmente con una tasa que depende de los coeficientes del problema. Además mostramos el modelamiento computacional del sistema mostrando los resultados obtenidos teóricamente.
\end{abstract}

Keywords. Ecuación Diferencial Parcial, viga, semigrupo, estabilidad polinomial.

\section{Abstract}

We studied the uniform stabilization of a class of Timoshenko systems with partial dissipation of the beam. Our main result is to prove that the semigroup associated to this model has polynomial decay. Moreover, we prove that the semigroup decays polynomially to zero. The system decays polynomially with rate depending on the coeficients of the problem. We also show the computational modeling of the system showing the results obtained theoretically.

Palabras clave. Differential partial equations, beam, semigroup, polynomial stability.

1. Introducción. Los estudios de las propiedades del sistema de Timoshenko fueron iniciadas en 1921 con el trabajo [1], siendo estudiado otras propiedades en [2, 3, 4], el cual fue considerando el siguiente problema:

$$
\begin{aligned}
\rho_{1} \varphi_{t t}-\kappa\left(\varphi_{x}+\psi\right)_{x} & =0, \quad(x, t) \in(0, L) \times(0, T), \\
\rho_{2} \psi_{t t}-b \psi_{x x}+\kappa\left(\varphi_{x}+\psi\right) & =0, \quad(x, t) \in(0, L) \times(0, T),
\end{aligned}
$$

donde $t$ es la variable temporal, $x$ la variable espacial definida en $(0, L)$, siendo $L$ la longitud de la viga. las funciones $\varphi, \psi$ representan el desplazamiento transversal y el ángulo de rotación de la sección transversal, respectivamente. Las constantes del sistema son definidas por $\rho_{1}=\rho A, \rho_{2}=\rho I, \kappa=K A G, b=E I$, donde $\rho$ denota la densidad, $A$ el área de la sección transversal, $I$ el área del momento de inercia, $K$ el coeficiente de cizalla $(K<1), E$ y $G$ representan constantes elásticas que dependen del material.

En este trabajo estamos interesados en obtener la estabilización del sistema $(1,1)-(1,2)$. Para ver otros casos de sistema de Timoshenko con disipación, ver [5, 6, 7].

\footnotetext{
* Laboratorio Nacional de Computación Científica, MCTIC, Petrópolis- Brasil.frankhaq@lncc.br.

$\dagger$ Universidad Peruana Cayetano Heredia, Universidad Nacional Mayor de San Marcos, Lima- Perú. neisser.pino@upch.pe, neisser.pino@unmsm.edu.pe.
} 
El objetivo de este trabajo es presentar de forma didáctica los resultados contenidos en el artículo [8], usando la teoría de semigrupos vista en [9] y también acrecentar los resultados de la parte numérica vista en [10] para nuestro trabajo que es el caso de disipación parcial.

2. El sistema de Timoshenko con disipación parcial. Vamos a considerar el siguiente problema

$$
\begin{aligned}
\rho_{1}(x) \varphi_{t t}-\left(\kappa(x)\left(\varphi_{x}+\psi\right)\right)_{x} & =0, \\
\rho_{2}(x) \psi_{t t}-\left(b(x) \psi_{x}\right)_{x}+\kappa(x)\left(\varphi_{x}+\psi\right) & =0 .
\end{aligned}
$$

Para este sistema vamos a considerar la condición de frontera en $x=0$

$$
\varphi(0, t)=\psi(0, t)=0,
$$

y condición de frontera en $x=L$ dada por

$$
\begin{aligned}
m \varphi_{t t}(L, t)+k_{0} \varphi_{t}(L, t)+\kappa(L)\left(\varphi_{x}(L, t)+\psi(L, t)\right) & =0 \\
I_{m} \psi_{t t}(L, t)+k_{1} \psi_{t}(L, t)+b(L) \psi_{x}(L, t) & =0 .
\end{aligned}
$$

y vamos a considerar el sistema sujeto a los siguientes datos iniciales

$$
\varphi(x, 0)=\varphi_{0}(x), \varphi_{t}(x, 0)=\varphi_{1}(x), \psi(x, 0)=\psi_{0}(x), \psi_{t}(x, 0)=\psi_{1}(x) .
$$

Los dos primeros términos de (2.4), $m \varphi_{t t}(L, t)+k_{0} \varphi_{t}(L, t)$, denotan la contribución de la inercia del cuerpo en la extremidad. Denotaremos $S(L, t)=\kappa(L)\left(\varphi_{x}(L, t)+\psi(L, t)\right)$, este término representa el amortecimiento proporcionado por el material granular, que es supuesto a ser proporcional al coeficiente de velocidad de amortecimiento $k_{0}$. El término $S(x, t)=\kappa(x)\left(\varphi_{x}(x, t)+\psi(x, t)\right)$ representa la fuerza de cizalla.

Donde $M(x, t)=b(x) \psi_{x}(x, t), M(L, t)=b(L) \psi_{x}(L, t)$ es el momento de flexión e $I_{m}$ es el momento de inercia de la extremidad.

En aplicaciones es importante reducir el mecanismo disipativo, como es el caso de nuestro trabajo. Así asumimos que las propiedades disipativas del sistema son producidas apenas por el momento de flexión de la viga. Con esto el modelo (2.1)-(2.6) se transforma en

$$
\begin{aligned}
\rho_{1} \varphi_{t t}-\kappa\left(\varphi_{x}+\psi\right)_{x} & =0, \\
\rho_{2} \psi_{t t}-b \psi_{x x}+\kappa\left(\varphi_{x}+\psi\right) & =0,
\end{aligned}
$$

con las condiciones de contorno en la extremidad derecha $x=L$

$$
\begin{aligned}
S(L, t)+\alpha \varphi(L, t) & =0, \\
M(L, t)+I_{m} \psi_{t t}(L, t)+k_{1} \psi_{t}(L, t) & =0,
\end{aligned}
$$

y para la extremidad izquierda $x=0$

$$
\begin{aligned}
\varphi(0, t) & =0, \\
M(0, t)-I_{m} \psi_{t t}(0, t)-k_{2} \psi_{t}(0, t) & =0,
\end{aligned}
$$

El sistema está sujeto a las condiciones iniciales (2.6).

Aqui asumimos que todos los coeficientes del modelo son constantes. Observe que el sistema (2.7)(2.12) no posee ningún mecanismo disipativo eficaz sobre la deflexión transversal.

2.1. Formulación abstracta. Primero vamos a mostrar la buena colocación del sistema (2.7)-(2.12). Para hazer esto vamos a denotar por $U=\left(\varphi, \varphi_{t}, \psi, \psi_{t}, v, w\right)^{\prime}$, entonces $U$ satisface el siguiente problema de Cauchy

$$
U_{t}=A_{1} U, \quad U(0)=U_{0}
$$

donde $U_{0}=\left(\varphi_{0}, \varphi_{1}, \psi_{0}, \psi_{1}, v_{0}, w_{0}\right)^{\prime}$ y $A_{1}: D\left(A_{1}\right) \subseteq H \rightarrow H$ es dado por

$$
A_{1}=\left(\begin{array}{cccccc}
0 & I & 0 & 0 & 0 & 0 \\
\frac{\kappa}{\rho_{1}} \partial_{x x} & 0 & \frac{\kappa}{\rho_{1}} \partial_{x} & 0 & 0 & 0 \\
0 & 0 & 0 & I & 0 & 0 \\
-\frac{\kappa}{\rho_{2}} \partial_{x} & 0 & \frac{b}{\rho_{2}} \partial_{x x}-\frac{\kappa}{\rho_{2}} & 0 & 0 & 0 \\
0 & 0 & -\frac{1}{I_{m}} \gamma_{3} & 0 & -\frac{k_{1}}{I_{m}} I & 0 \\
0 & 0 & \frac{1}{I_{m}} \gamma_{4} & 0 & 0 & \frac{k_{2}}{m} I
\end{array}\right),
$$


donde $\gamma_{3}(\psi)=b \psi_{x}(L)$, y $\gamma_{4}(\psi)=b \psi_{x}(0)$. Por $H$ denotamos el espacio complejo

$$
H=H_{*}^{1}(0, L) \times L^{2}(0, L) \times H^{1}(0, L) \times L^{2}(0, L) \times \mathbb{C}^{2},
$$

donde $H_{*}^{1}(0, L)=\left\{w \in H^{1}(0, L) \mid w(0, L)=0\right\}$.

Vamos a dotar el espacio $H$ con el siguiente producto interno: con

$$
\begin{aligned}
\left(U_{1}, U_{2}\right)_{H}= & \int_{0}^{L} \rho_{1} \Phi_{1} \overline{\Phi_{2}} d x+\int_{0}^{L} \rho_{2} \Psi_{1} \overline{\Psi_{2}} d x+\int_{0}^{L} b\left(\psi_{1}\right)_{x} \overline{\left(\psi_{2}\right)_{x}} d x \\
& +\int_{0}^{L} \kappa\left(\left(\varphi_{1}\right)_{x}+\psi_{1}\right) \overline{\left(\left(\varphi_{2}\right)_{x}+\psi_{2}\right)} d x+\alpha \varphi_{1}(L) \overline{\varphi_{2}(L)}+I_{m} v_{1}(L) v_{2}(L)+I_{m} w_{1}(L) w_{2}(L)
\end{aligned}
$$

donde $U_{j}=\left(\varphi_{j}, \Phi_{j}, \psi_{j}, \Psi_{j}, v_{j}, w_{j}\right)^{\prime} \in H, j=1,2, \Phi_{j}=\left(\varphi_{j}\right)_{t} \mathrm{y} \Psi_{j}=\left(\psi_{j}\right)_{t}$. Este produto interno induce una norma (equivalente) en $H$ dada por

$$
\begin{aligned}
\|U\|_{H}^{2}= & \int_{0}^{L}\left[\rho_{1}|\Phi|^{2}+\rho_{2}|\Psi|^{2}+b\left|\psi_{x}\right|^{2}+\kappa\left|\varphi_{x}+\psi\right|^{2}\right] d x \\
& +\alpha|\varphi(L)|^{2}+I_{m}|v|^{2}+I_{m}|w|^{2} .
\end{aligned}
$$

donde $U=(\varphi, \Phi, \psi, \Psi, v, w)^{\prime} \operatorname{con} \Phi=\varphi_{t} \mathrm{y} \Psi=\psi_{t}$.

Por tanto el domíno del operador $A_{1}$ será, con $S(L, t)=S(L)$ para simplicidad en lo que sigue

$$
D\left(A_{1}\right)=\left\{U \in H \mid A_{1} U \in H, S(L)+\alpha \varphi(L)=0, \Psi(L)=v, \Psi(0)=w\right\} .
$$

La ecuación resolvente es dada al resolver $i \lambda U-A_{1} U=F$, donde $U=(\varphi, \Phi, \psi, \Psi, v, w)^{\prime} \in H \mathrm{y}$ $F=\left(f_{1}, f_{2}, f_{3}, f_{4}, f_{5}, f_{6}\right)^{\prime} \in H$ y $t \in R$. En términos de las componentes la ecuación resolvente es escrita como sigue

$$
\begin{aligned}
\lambda \varphi-\Phi & =f_{1}, \\
\lambda \rho_{1} \Phi-k\left(\varphi_{x}+\psi\right)_{x} & =f_{2}, \\
\lambda \psi-\Psi & =f_{3}, \\
\lambda \rho_{2} \Psi-b \psi_{x x}+\kappa\left(\varphi_{x}+\psi\right) & =f_{4}, \\
I_{m} \lambda v+k_{1} v+b \psi_{x}(L) & =f_{5}, \\
I_{m} \lambda w+k_{2} w+b \psi_{x}(0) & =f_{6}, \\
S(L)+\alpha \varphi(L)=0, \quad \varphi(0) & =0 .
\end{aligned}
$$

Prosiguiendo con los cálculos tenemos

$$
R e\left(A_{1} U, U\right)_{H}=-k_{1}|v|^{2}-k_{2}|w|^{2} \leq 0
$$

y así

$$
k_{1}|v|^{2}+k_{2}|w|^{2}=\operatorname{Re}(F, U)_{H}
$$

donde $F=\left(f_{1}, f_{2}, f_{3}, f_{4}, f_{5}, f_{6}\right)$.

El principal resultado de esta Sección es dado por el siguiente Teorema.

Teorema 1. El operador $A_{1}$ es generador infinitesimal del semigrupo de contracciones $C_{0}$. Demostración: Como en [8], y en forma análoga de los Lemas 3.2.1 y 3.2.3 de [10], tenemos que $A_{1}$ es definido denso y disipativo. Resta mostrar que $0 \in \rho\left(A_{1}\right)$, es decir, probar que existe una única solución del sistema (2.16)-(2.22) para $\lambda=0$. Así, tenemos el sistema (2.16)-(2.19) anulando los términos que tienen a $\lambda$, se obtiene, $-\Phi=f_{1},-\Psi=f_{3}$. Tenemos las siguientes igualdades

$$
\begin{aligned}
-\kappa\left(\varphi_{x}+\psi\right)_{x} & =f_{2}, \\
-b \psi_{x x}+\kappa\left(\varphi_{x}+\psi\right) & =f_{4},
\end{aligned}
$$

con las siguientes condiciones de contorno

$$
\varphi(0)=S(L)+\alpha \varphi(L)=0, \quad b \psi_{x}(0)=f_{6}+k_{2} f_{3}(0), \quad b \psi_{x}(L)=f_{5}+k_{1} f_{3}(L) .
$$


El problema definido es bien colocado. Con efecto, denotamos por $m(x)$, la función, $m(x)=A x^{2}+B x$, donde $A$ y $B$ son tales que

$$
A=b m_{x}(0)=b \psi_{x}(0), \quad B=b m_{x}(L)=b \psi_{x}(L) .
$$

Denotamos por $\widetilde{\psi}=\psi-m(x)$ el sistema de encima puede ser escrito como

$$
\begin{gathered}
-\left[\kappa\left(\varphi_{x}+\widetilde{\psi}\right)\right]_{x}=f, \\
-b \widetilde{\psi}_{x x}+\kappa\left(\varphi_{x}+\widetilde{\psi}\right)=g, \\
\varphi(0)=S(L)+\alpha \varphi(L)=0, \quad b \widetilde{\psi}_{x}(0)=0, \quad b \widetilde{\psi}_{x}(L)=0,
\end{gathered}
$$

con $f=f_{2}-\kappa m_{x}$ y $g=f_{4}+b m_{x x}-\kappa m$. Para mostrar la buena colocación del problema, vamos a considerar el espacio de Hilbert $V=H_{0}^{1}(0, L) \times H^{1}(0, L)$ y la forma bilineal

$$
a\left(\varphi^{1}, \varphi^{2}, \psi^{1}, \psi^{2}\right)=\int_{0}^{L} \kappa\left(\varphi_{x}^{1}+\psi^{1}\right) \overline{\left(\varphi_{x}^{2}+\psi^{2}\right)}+b \psi_{x}^{1} \overline{\psi_{x}^{2}} d x+\alpha \varphi^{1}(L) \varphi^{2}(L) .
$$

- $a(\cdot, \cdot)$ es bilineal;

- $a(\cdot, \cdot)$ es continual, la prueba de estos resultados es análoga a la vista en las páginas 26 y 27 de [10]

- $a(\cdot, \cdot)$ es coerciva. Para verificar la coercividad, vamos a considerar la identidad

$$
-\kappa \varphi_{x x}=\kappa \psi_{x}-\kappa\left(\varphi_{x x}+\psi_{x}\right)
$$

y multiplicando por $\bar{\varphi}$ obtenemos

$$
\alpha \varphi(L)^{2}+\kappa \bar{\varphi}(L) \psi(L)+\kappa \int_{0}^{L}\left|\varphi_{x}\right|^{2} d x=-\kappa \int_{0}^{L} \psi_{x} \bar{\varphi} d x+\kappa \int_{0}^{L}\left(\varphi_{x}+\psi\right) \bar{\varphi}_{x} d x+\alpha \varphi(L)^{2} .
$$

Usando la desigualdad de Poincaré, existe una constante $C>0$ tal que

$$
\int_{0}^{L}\left|\varphi_{x}\right|^{2} d x \leq C \int_{0}^{L}\left|\psi_{x}\right|^{2} d x+c \int_{0}^{L}\left(\varphi_{x}+\psi\right)^{2} d x+C_{\epsilon}|\varphi(L)|^{2}+\epsilon|\psi(L)|^{2} .
$$

Por otro lado, note que

$$
\begin{aligned}
\int_{0}^{L}|\psi|^{2} d x & =\int_{0}^{L}\left(\varphi_{x}+\psi\right)^{2} d x-\int_{0}^{L}\left|\varphi_{x}\right|^{2} d x-2 \operatorname{Re} \int_{0}^{L} \varphi_{x} \bar{\psi} d x \\
& \leq \int_{0}^{L}\left(\varphi_{x}+\psi\right)^{2} d x+c \int_{0}^{L}\left|\varphi_{x}\right|^{2} d x+\frac{1}{2} \int_{0}^{L}|\psi|^{2} d x .
\end{aligned}
$$

Entonces

$$
\int_{0}^{L}|\psi|^{2} d x \leq c \int_{0}^{L}\left(\varphi_{x}+\psi\right)^{2} d x+c \int_{0}^{L}\left|\varphi_{x}\right|^{2} d x .
$$

Usando (2.30) y la Desigualdad de Poincaré, obtenemos

$$
\int_{0}^{L}|\psi|^{2}+|\varphi|^{2} d x \leq C \int_{0}^{L}\left(\varphi_{x}+\psi\right)^{2} d x+C \int_{0}^{L}|\psi|^{2} d x+C_{\epsilon}|\varphi(L)|^{2}+\epsilon|\psi(L)|^{2} .
$$

Desde

$$
|\psi(L)|^{2} \leq C \int_{0}^{L}|\psi|^{2}+\left|\psi_{x}\right|^{2} d x
$$

para $\epsilon$ suficientemente pequeño, obtenemos

$$
\int_{0}^{L}|\psi|^{2}+|\varphi|^{2} d x \leq C \int_{0}^{L}\left(\varphi_{x}+\psi\right)^{2} d x+C \int_{0}^{L}|\psi|^{2} d x+C_{\epsilon}|\varphi(L)|^{2} .
$$

Por el Lema de Lax-Milgram la buena colocación se obtiene. En particular $0 \in \rho\left(A_{1}\right)$ y por el Corolario 2.7.1 de [10], tenemos que $A_{1}$ genera un semigrupo de clase $C_{0}$ de contracciones. 
2.2. Resultados Auxiliares. Mostraremos los siguientes resultados que nos ayudarán en el desarrollo de nuestro trabajo.

Teorema 2 (Borichev Tomilov). Sea $\{T(t)\}$ un semigrupo de clase $C_{0}$ en un espacio de Hilbert $H$ con generador infinitesimal $A_{1}$, tal que, $i \mathbb{R} \subset \rho\left(A_{1}\right)$. Entonces las siguientes desigualdades son equivalentes

$$
\frac{1}{|\eta|^{\alpha}}\left\|\left(i \eta-A_{1}\right)^{-1}\right\| \leq C, \forall \eta \in \mathbb{R}
$$

$y$

$$
\left\|T(t) A_{1}^{-1}\right\| \leq \frac{c}{t^{\frac{1}{\alpha}}}
$$

Con el objetivo de estabilizar el sistema con el mínimo de disipación, fue introducido un nuevo criterio, conocido como igualdad de velocidad de las ondas, que es caracterizada por la siguiente identidad.

$$
\chi=\frac{\rho_{1}}{\rho_{2}}-\frac{\kappa}{b} .
$$

En términos de las componentes la ecuación resolvente del sistema de Timoshenko (1.1)-(1.2) es escrita como

$$
\begin{aligned}
\lambda \varphi-\Phi & =f_{1} \\
\lambda \rho_{1} \Phi-\left[\kappa(x)\left(\varphi_{x}+\psi\right)\right]_{x} & =f_{2} \\
\lambda \psi-\Psi & =f_{3}, \\
\lambda \rho_{2} \Psi-\left[b(x) \psi_{x}\right]_{x}+\kappa(x)\left(\varphi_{x}+\psi\right) & =f_{4}, \\
m \lambda u+k_{0} u+\kappa(L)\left(\varphi_{x}+\psi\right)(L) & =f_{5}, \\
I_{m} \lambda v+k_{1} v+b(L) \psi_{x}(L) & =f_{6} .
\end{aligned}
$$

Ahora vamos a introducir algunas notaciones.

$$
\begin{gathered}
I_{\varphi}(\beta)=\rho_{1} q_{1} \kappa|\Phi(\beta)|^{2}+q_{1}\left|\kappa\left(\varphi_{x}(\beta)+\psi(\beta)\right)\right|^{2}, \\
I_{\psi}(\beta)=\rho_{2} q_{2} b|\Psi(\beta)|^{2}+q_{2}\left|b \psi_{x}(\beta)\right|^{2}, \\
N^{2}=\int_{0}^{L}\left[\rho_{1}|\Phi|^{2}+\rho_{2}|\Psi|^{2}+b\left|\psi_{x}\right|^{2}+\kappa\left|\varphi_{x}+\psi\right|^{2}\right] d x .
\end{gathered}
$$

donde $q_{1}$ y $q_{2}$ son funciones continuas.

Con las notaciones enunciadas, tenemos el siguiente Lema

Lema 1. Suponemos que $(\varphi, \Phi, \psi, \Psi, u, v)$ sea una solución fuerte para el sistema (2.34)- (2.39). Entonces existe una constante positiva $C$ tal que

$$
N^{2} \leq C\left[I_{\varphi}(\beta)+I_{\psi}(\beta)+\|F\|_{H}^{2}\right]
$$

$y$

$$
I_{\varphi}(\beta)+I_{\psi}(\beta) \leq C\left[N^{2}+\|F\|_{H}^{2}\right]
$$

para $\beta=0, L$.

3. Decaimiento Polinomial. El objetivo de esta sección es demostrar el siguiente teorema.

Teorema 3. El semigrupo $T(t): H \rightarrow H$ definido por el sistema de Timoshenko $(2,7)-(2,12)$ decae polinomialmente, es decir,

$$
\left\|T(t) U_{0}\right\| \leq \frac{C}{t^{\frac{1}{2}}}\left\|U_{0}\right\|_{D\left(A_{1}\right)},
$$

si $\chi=0$, donde $\chi$ es dado en (2.33). Por otro lado, si $\chi \neq 0$

$$
\left\|T(t) U_{0}\right\| \leq \frac{C}{t^{\frac{1}{4}}}\left\|U_{0}\right\|_{D\left(A_{1}\right)},
$$


donde $T(t) U_{0}$ es el semigrupo junto con la condición inicial del problema de Cauchy (2.13), asociado a nuestro problema (2.7)-(2.12).

Demostración: Vamos a mostrar que $i \mathbb{R} \subset \rho\left(A_{1}\right)$, de manera similar al Teorema 3.3.1 de [10] y que la desigualdad dada en el Teorema 2, que está en el trabajo [11] es válida con $\alpha=2$, si $\chi=0$ y con $\alpha=4$, si $\chi \neq 0$.

Paso 1: $i \mathbb{R} \subset \rho\left(A_{1}\right)$. Supongamos que hay un valor propio imaginario $i \lambda U-A_{1} U=0$. Con efecto, usando (2.23), tenemos que $v=w=0$. Para las relaciones (2.18)-(2.21) y haciendo $F=0$, tenemos que $\psi$ satisface

$$
\psi_{x x}+\alpha^{2} \psi=-\frac{\kappa}{b} \varphi_{x}, \text { donde } b \alpha^{2}=\lambda^{2} \rho_{2}-\kappa
$$

con condiciones de contorno

$$
\psi(0)=\psi_{x}(0)=\psi(L)=\psi_{x}(L)=0
$$

Multiplicando la ecuación (2.25) por $\bar{\psi}_{x}$, integrando sobre $(0, L)$ y tomando la parte real, sigue que

$$
\operatorname{Re}\left\{\int_{0}^{L} \varphi_{x} \bar{\psi}_{x} d x\right\}=0
$$

Multiplicando las ecuaciones (2.18) y (2.19) por $\bar{\psi}$, integrando sobre $(0, L)$ y tomando la parte real, obtenemos que

$$
\operatorname{Re}\left\{\int_{0}^{L} \Phi \bar{\Psi} d x\right\}=0 \Rightarrow \operatorname{Re}\left\{\int_{0}^{L} \varphi \bar{\psi} d x=0\right\} .
$$

Multiplicando la equación (3.3) por $\bar{\varphi}$ integrando sobre $(0, L)$ y usando (3.4) y (3.5), obtenemos que $\varphi(L)=0$. Por tanto, de las condiciones de contorno (2.9)-(2.12) obtenemos que $\varphi_{x}(L)=0$. Del Lema 1 aplicado a $\beta=L$ concluimos que $\varphi=\psi=0$. Entonces $U=0$ el qual es una contradicción. Por lo tanto no hay valores propios imaginarios.

Paso 2: Valen las siguientes estimativas

$$
\frac{1}{|\lambda|^{2}}\left\|\left(\lambda_{i} I-A_{1}\right)^{-1}\right\|_{H} \leq C, \forall \lambda \in \mathbb{R}, \text { si } \chi=0
$$

y

$$
\frac{1}{|\lambda|^{4}}\left\|\left(\lambda_{i} I-A_{1}\right)^{-1}\right\|_{H} \leq C, \forall \lambda \in \mathbb{R}, \text { si } \chi \neq 0 .
$$

Multiplicando la ecuación (2.17) por $\bar{\psi},(2.19)$ por $\overline{\psi_{x}}$ obtenemos

$$
\begin{array}{r}
i \rho_{1} \lambda \Phi \bar{\psi}-\kappa \varphi_{x x} \bar{\psi}-\kappa \psi_{x} \bar{\psi}=f_{2} \bar{\psi}, \\
i \rho_{2} \lambda \Psi \bar{\psi}_{x}-b \psi_{x x} \bar{\psi}_{x}+\kappa \varphi_{x} \bar{\psi}_{x}+\kappa \psi \bar{\psi}_{x}=f_{4} \bar{\psi}_{x} .
\end{array}
$$

Integrando por partes, tomando la parte real y usando (2.9) obtenemos

$$
\begin{gathered}
\operatorname{Re} \int_{0}^{L} i \rho_{1} \lambda \Phi \bar{\psi} d x+\kappa \operatorname{Re} \int_{0}^{L} \varphi_{x} \bar{\psi}_{x} d x+\operatorname{Re} A_{\psi}=\operatorname{Re} \int_{0}^{L} f_{2} \bar{\psi} d x \\
\operatorname{Re} \int_{0}^{L} \kappa \varphi_{x} \bar{\psi}_{x} d x+B_{\psi}=\operatorname{Re} \int_{0}^{L} f_{4} \bar{\psi} d x
\end{gathered}
$$

donde

$$
\begin{gathered}
A_{\psi}=\frac{\kappa}{2}|\psi(L)|^{2}+\frac{\kappa}{2}|\psi(0)|^{2}+\kappa \varphi_{x}(0) \bar{\psi}(0)+\alpha \operatorname{Re} \varphi(L) \bar{\psi}(L), \\
B_{\psi}=\rho_{2}|\Psi(L)|^{2}-\rho_{2}|\Psi(0)|^{2}+b\left|\psi_{x}(L)\right|^{2}-b\left|\psi_{0}(L)\right|^{2}+\kappa|\psi(L)|^{2}-\kappa|\psi(0)|^{2} .
\end{gathered}
$$


De las condiciones de contorno de $\psi$ y de la relación (2.24) obtenemos

$$
\left|B_{\psi}\right| \leq c|\lambda|^{2}\|U\|\|F\| .
$$

Usando el Lema 1 obtenemos

$$
\begin{aligned}
\left|A_{\psi}\right| & \leq \alpha|\varphi(L) \bar{\psi}(L)|+\left|\kappa \varphi_{x}(0) \bar{\psi}(0)\right|+c\|U\|\|F\| \\
& \leq \alpha|\varphi(L) \bar{\psi}(L)|+c\|U\||\bar{\psi}(0)|+c\|U\|\|F\|
\end{aligned}
$$

para $\lambda$ suficientemente grande. Por tanto, de (3.11) tenemos que

$$
\left|\operatorname{Re} \int_{0}^{L} \varphi_{x} \bar{\psi}_{x} d x\right| \leq c|\lambda|^{2}\|U\|\|F\|
$$

para $\lambda$ suficientemente grande. Usando la ecuación de arriba (3.10), obtenemos que

$$
\left|\operatorname{Re} \int_{0}^{L} \Phi_{x} \bar{\Psi} d x\right| \leq c|\lambda|^{2}\|U\|\|F\|+c\|U\||\bar{\psi}(0)|+\alpha x|\varphi(L) \bar{\psi}(L)|,
$$

lo cual implica que

$$
\left|\operatorname{Re} \int_{0}^{L} \phi_{x} \bar{\psi} d x\right| \leq c\|U\|\|F\|+\frac{\alpha}{|\lambda|^{2}}|\varphi(L) \bar{\psi}(L)|+\frac{c}{|\lambda|^{2}}\|U\||\bar{\psi}(0)|+c\|F\|^{2} .
$$

Multiplicando las ecuaciones (2.34) y (2.35) por $\bar{\psi}$ y tomando la parte real, obtenemos

$$
-\operatorname{Re} \int_{0}^{L} \Phi \bar{\Psi} d x+\frac{k}{\rho_{1}} \operatorname{Re} \int_{0}^{L} \varphi_{x} \bar{\psi}_{x} d x=R_{6} .
$$

Multiplicando las ecuaciones (2.36) y (2.37) por $\bar{\varphi}$ y tomando la parte real, obtenemos

$$
-\operatorname{Re} \int_{0}^{L} \Psi \bar{\Phi} d x+\frac{b}{\rho_{2}} \operatorname{Re} \int_{0}^{L} \psi_{x} \bar{\varphi}_{x} d x+\frac{\kappa}{\rho_{2}}|\varphi(L)|^{2}+\frac{\kappa}{\rho_{2}} \operatorname{Re} \int_{0}^{L} \psi \bar{\varphi} d x=R_{7}
$$

donde $R_{i}$ para $i=6,7$ es tal que

$$
\left|R_{i}\right| \leq c\|U\|\|F\| .
$$

Tomando la diferencia de (3.16) con (3.17) y usando $\frac{\kappa}{\rho_{1}}=\frac{b}{\rho_{2}}$, obtenemos

$$
|\varphi(L)|^{2} \leq c\|U\|\|F\|+\frac{\alpha}{|\lambda|^{2}}|\varphi(L) \bar{\psi}(L)|+\frac{c}{|\lambda|^{2}}\|U\||\bar{\psi}(0)|+c\|F\|^{2},
$$

lo cual implica que

$$
|\Phi(L)|^{2} \leq c|\lambda|^{2}\|U\|\|F\|+c\|U\||\bar{\psi}(0)|+c|\lambda|^{2}\|F\|^{2} .
$$

De (2.22) tenemos también

$$
\left|\varphi_{x}(L)\right|^{2} \leq c|\lambda|^{2}\|U\|\|F\|+c\|U\||\bar{\psi}(0)|+c|\lambda|^{2}\|F\|^{2} .
$$

Usando el Lema 1 para $\beta=L$ obtenemos

$$
\int_{0}^{L}|\Psi|^{2}+\left|\psi_{x}\right|^{2}+|\Phi|^{2}+\left|\varphi_{x}\right|^{2} d s \leq c I_{\psi}(L)+c I_{\phi}(L)+c\|U\|\|F\| .
$$

Entonces

$$
\int_{0}^{L}|\Psi|^{2}+\left|\psi_{x}\right|^{2}+|\Phi|^{2}+\left|\varphi_{x}\right|^{2} d s \leq c|\lambda|^{2}\|U\|\|F\|
$$

y para $\lambda$, suficientemente grande,

$$
\|U\|^{2} \leq c|\lambda|^{4}\|F\|^{2} .
$$


Ahora vamos a probar (3.7) con $\chi \neq 0$. De la identidad (3.17) y de las desigualdades (3.13)-(3.15) obtenemos

$$
|\varphi(L)|^{2} \leq c|\lambda|^{2}\|U\|\|F\|+c\|F\|^{2}+\alpha|\varphi(L) \bar{\psi}(L)|+c\|U\||\bar{\psi}(0)|
$$

lo cual implica

$$
|\Phi(L)|^{2} \leq c|\lambda|^{4}\|U\|\|F\|+c|\lambda|^{2}\|F\|^{2}+c\|U\||\bar{\Psi}(0)| .
$$

De (2.22) tenemos también

$$
\left|\varphi_{x}(L)\right|^{2} \leq c|\lambda|^{4}\|U\|\|F\|+c|\lambda|^{2}\|F\|^{2}+c\|U\||\bar{\Psi}(0)| .
$$

Para $\lambda$ grande. Entonces usando el Lema 1 obtenemos

$$
\int_{0}^{L}|\Psi|^{2}+\left|\psi_{x}\right|^{2}+|\Phi|^{2}+\left|\varphi_{x}\right|^{2} d s \leq c I_{\psi}(L)+c I_{\phi}(L)+c\|U\|\|F\| .
$$

Usando el mismo raciocinio usado arriba

$$
\int_{0}^{L}|\Psi|^{2}+\left|\psi_{x}\right|^{2}+|\Phi|^{2}+\left|\varphi_{x}\right|^{2} d s \leq c|\lambda|^{4}\|U\|\|F\|
$$

y para $\lambda$, suficientemente grande,

$$
\|U\|^{2} \leq c|\lambda|^{8}\|F\|^{2} .
$$

Paso 3: Conclusión: Sigue del Teorema de Borichev-Tomilov dado en el Teorema 2, que está en el trabajo [11]. Con esto tenemos la prueba del decaimiento polinomial del semigrupo generado por (2.7)-(2.12).

Obervación 1 (Falta de estabilidad exponencial). El semigrupo definido por el sistema de Timoshenko (2.7)-(2.12) no tiene decaimiento exponencial, la prueba de este resultado es análoga a la prueba del Teorema 3.4.1 presentado en [10].

4. Abordaje Computacional y Discretización del Sistema. Realizamos la discretización del sistema (2.7)-(2.12), usando diferencias finitas para las variables temporal y espacial, para las derivadas de orden 2 usamos diferencia centrada y para las derivadas de orden 1 usamos el método explícito.

Obervación 2. Para nuestro análisis, los coeficientes serán constantes.

La discretización del tiempo es dada por

$$
t_{0}=0<t_{1}=\Delta t<\ldots<t_{n}<\ldots<t_{N}=N \Delta t<t_{N+1}=T
$$

donde $t_{n}=n \Delta t$ para $n=0,1,2, \ldots N+1 \mathrm{y}$ hacemos

$$
h_{x}=\Delta x=\frac{L}{i+1}, \Delta t=\frac{T}{N+1}, L, T \in \mathbb{N}
$$

con $x_{i}=i \Delta x, i=0,1,2, \ldots, I+1$.

Consideremos también los siguientes operadores de diferencias finitas en espacio y en el tiempo:

Esquema Progresivo Implícito (primer orden):

$$
\partial_{x} u_{j}^{n}=\frac{u_{j+1}^{n}-u_{j}^{n}}{\Delta x}, \quad \partial_{t} u_{j}^{n}=\frac{u_{j}^{n+1}-u_{j}^{n}}{\Delta t}
$$

Esquema Atrasado Explícito (primer orden):

$$
\bar{\partial}_{x} u_{j}^{n}=\frac{u_{j}^{n}-u_{j-1}^{n}}{\Delta x}, \quad \bar{\partial}_{t} u_{j}^{n}=\frac{u_{j}^{n}-u_{j}^{n-1}}{\Delta t}
$$

Diferencia Central (segundo orden):

$$
\frac{\partial_{x}+\bar{\partial}_{x}}{2} u_{j}^{n}=\frac{u_{j+1}^{n}-u_{j-1}^{n}}{2 \Delta x}, \quad \frac{\partial_{t}+\bar{\partial}_{t}}{2} u_{j}^{n}=\frac{u_{j}^{n+1}-u_{j}^{n-1}}{2 \Delta t}
$$

Esquema de Diferencias Centrada (segundo orden)

$$
\bar{\partial}_{x} \partial_{x} u_{j}^{n}=\frac{u_{j+1}^{n}-2 u_{j}^{n}+u_{j-1}^{n}}{\Delta x^{2}}, \quad \bar{\partial}_{t} \partial_{t} u_{j}^{n}=\frac{u_{j}^{n+1}-2 u_{j}^{n}+u_{j}^{n-1}}{\Delta t^{2}},
$$

En todos los casos $u_{j}^{n}$ corresponde a la solución numérica en los puntos nodales $\left(x_{j}, t_{n}\right)$ de la discretización, y tales operadores son construidos con base en la aplicación de la serie de Taylor para $u\left(x_{j}, t_{n}\right)$. Para otros esquemas numéricos ver [12]. 
4.1. Convergencia del Esquema Numérico. Tenemos la siguiente definición:

Definición 1. Decimos que un esquema numérico en diferencias finitas expresado por

$F_{(\Delta x, \Delta t)} u(\Delta x, \Delta t)=0$ y que aproxima una equación diferencial parcial $F u(x, t)=0$, es convergente en cada punto $\left(x_{j}, t_{n}\right)$ si para $\left(x_{j}, t_{n}\right) \longrightarrow(x, t)$ tenemos que $u_{j}^{n} \rightarrow u$ cuando $\Delta x, \Delta t \rightarrow 0$. Formalmente

$$
\lim _{\Delta x \rightarrow 0} \operatorname{máx}_{j=0,1,2, \ldots} \lim _{\Delta t \rightarrow 0} \operatorname{máx}_{n=0,1,2, \ldots}\left|u\left(x_{j}, t_{n}\right)-u_{j}^{n}\right|=0
$$

Así nuestro sistema queda expresado de la siguiente manera

$$
\begin{aligned}
\rho_{1}\left(\frac{\varphi_{i}^{n+1}-2 \varphi_{i}^{n}+\varphi_{i}^{n-1}}{\Delta t^{2}}\right)-\kappa\left(\frac{\varphi_{i+1}^{n}-2 \varphi_{i}^{n}+\varphi_{i-1}^{n}}{h_{x}^{2}}+\frac{\psi_{i}^{n}-\psi_{i-1}^{n}}{h_{x}}\right) & =0, \\
\rho_{2}\left(\frac{\psi_{i}^{n+1}-2 \psi_{i}^{n}+\psi_{i}^{n-1}}{\Delta t^{2}}\right)-b\left(\frac{\psi_{i+1}^{n}-2 \psi_{i}^{n}+\psi_{i-1}^{n}}{h_{x}^{2}}\right)+\kappa\left(\frac{\varphi_{i}^{n}-\varphi_{i-1}^{n}}{h_{x}}\right)-\kappa \psi_{i}^{n} & =0 .
\end{aligned}
$$

De (4.1) tenemos

$$
\frac{\rho_{1}}{\Delta t^{2}} \varphi_{i}^{n+1}-\frac{2 \rho_{1}}{\Delta t^{2}} \varphi_{i}^{n}+\frac{\rho_{1}}{\Delta t^{2}} \varphi_{i}^{n-1}=\frac{\kappa}{h_{x}^{2}} \varphi_{i+1}^{n}-\frac{2 \kappa}{h_{x}^{2}} \varphi_{i}^{n}+\frac{\kappa}{h_{x}^{2}} \varphi_{i-1}^{n}+\frac{\kappa}{h_{x}} \psi_{i}^{n}-\frac{\kappa}{h_{x}} \psi_{i-1}^{n} .
$$

De (4.2) tenemos

$$
\frac{\rho_{2}}{\Delta t^{2}} \psi_{i}^{n+1}-\frac{2 \rho_{2}}{\Delta t^{2}} \psi_{i}^{n}+\frac{\rho_{2}}{\Delta t^{2}} \psi_{i}^{n-1}=\frac{b}{h_{x}^{2}} \psi_{i+1}^{n}-\frac{2 b}{h_{x}^{2}} \psi_{i}^{n}+\frac{b}{h_{x}^{2}} \psi_{i-1}^{n}-\frac{\kappa}{h_{x}} \varphi_{i-1}^{n}+\frac{\kappa}{h_{x}} \varphi_{i-1}^{n}-\kappa \psi_{i}^{n} .
$$

La condición de contorno en $x=0$ es escrita como:

$$
\begin{aligned}
\varphi_{i}^{n} & =0 \\
b\left(\frac{\psi_{i}^{n}-\psi_{i-1}^{n}}{h_{x}}\right)-I_{m}\left(\frac{\psi_{i}^{n+1}-2 \psi_{i}^{n}+\psi_{i}^{n-1}}{\Delta t^{2}}\right)-k_{2}\left(\frac{\psi_{i}^{n}-\psi_{i}^{n-1}}{\Delta t}\right) & =0
\end{aligned}
$$

De (4.4) tenemos

$$
\frac{b}{h_{x}} \psi_{i}^{n}-\frac{b}{h_{x}} \psi_{i-1}^{n}-\frac{I_{m}}{\Delta t^{2}} \psi_{i}^{n+1}+\frac{2 I_{m}}{\Delta t^{2}} \psi_{i}^{n}-\frac{I_{m}}{\Delta t^{2}} \psi_{i}^{n-1}-\frac{k_{2}}{\Delta t} \psi_{i}^{n}+\frac{k_{2}}{\Delta t} \psi_{i}^{n-1}=0
$$

La condición de contorno en $x=L$ es escrita como:

$$
\begin{aligned}
\kappa\left(\frac{\varphi_{i}^{n}-\varphi_{i-1}^{n}}{h_{x}}+\psi_{i}^{n}\right)+\alpha \varphi_{i}^{n} & =0 \\
b\left(\frac{\psi_{i}^{n}-\psi_{i-1}^{n}}{h_{x}}\right)+I_{m}\left(\frac{\psi_{i}^{n+1}-2 \psi_{i}^{n}+\psi_{i}^{n-1}}{\Delta t^{2}}\right)+k_{1}\left(\frac{\psi_{i}^{n}-\psi_{i}^{n-1}}{\Delta t}\right) & =0
\end{aligned}
$$

De (4.5) tenemos

$$
\frac{\kappa}{h_{x}} \varphi_{i}^{n}-\frac{\kappa}{h_{x}} \varphi_{i-1}^{n}+\kappa \psi_{i}^{n}+\alpha \varphi_{i}^{n}=0
$$

De (4.6) tenemos

$$
\frac{b}{h_{x}} \psi_{i}^{n}-\frac{b}{h_{x}} \psi_{i-1}^{n}+\frac{I_{m}}{\Delta t^{2}} \psi_{i}^{n+1}-\frac{2 I_{m}}{\Delta t^{2}} \psi_{i}^{n}+\frac{I_{m}}{\Delta t^{2}} \psi_{i}^{n-1}+\frac{k_{1}}{\Delta t} \psi_{i}^{n}-\frac{k_{1}}{\Delta t} \psi_{i}^{n-1}=0
$$

4.2. Consistencia y Estabilidad. En particular, el concepto de consistencia nos dice que la solución exacta de un problema de valor inicial y de contorno satisface las ecuaciones de diferencias de los métodos numéricos con un margen de error dado, que se obtiene de la aproximación via serie de Taylor.

Definición 2. Para $F u(x, t)=0$ y $F_{(\Delta x, \Delta t)} u(\Delta x, \Delta t)=0$, decimos que el esquema numérico es consistente si para cualquier función $u=u(x, t)$ suficientemente regular se tiene que:

$$
F_{(\Delta x, \Delta t)} u(\Delta x, \Delta t) \rightarrow 0 \text { cuando }(\Delta x, \Delta t) \rightarrow 0
$$

Definición 3. Un método de diferencias finitas es estable si existe una constante $M>0$, tal que, $\left|e_{j}^{n}\right|<M$ para todo $j, n$. Donde $e_{j}^{n}$ es el error del método usado.

Teorema 4 (Convergencia Numérica). El esquema numérico explícito espacio-tiempo aplicado al sistema, es convergente sí, y solamente sí,

$$
\Delta t \leq \frac{\Delta x}{c}
$$


donde $c=\sqrt{E / \rho}$, $\rho$ es la densidad.

Demostración: Ver [13, 14].

Obervación 3 (Propiedades de la Energía Discreta del Método Explícito). Para la discretización de la energía usaremos también diferencias finitas en la variable espacial $x$.

4.3. Resultados Computacionales. A continuación escribiremos los resultados de la simulación numérica realizados con el método explícito de diferencias finitas, principalmente lo que nos muestra es la falta de decaimiento exponencial de la energía. Tratándose de un modelo de dimensión finita, se debe determinar los parámetros adecuados para asegurar buenos resultados, en este caso usamos los parametros dados en [10].

Por lo tanto consideramos el caso con una viga de longitud $L=1$, con las siguientes constantes físicas $E=1 \times 10^{4} \mathrm{~N} / \mathrm{m}^{2}, G=4,12 \times 10^{4} \mathrm{~N} / \mathrm{m}^{2}, \rho=1500 \mathrm{~kg} / \mathrm{m}^{3}$ y $\mathrm{K}=2 / 6$. Con las siguientes condiciones iniciales $\varphi(x, 0)=\psi(x, 0)=8 \operatorname{sen}(4 \pi x), \varphi_{t}(x, 0)=\psi_{t}(x, 0)=0$.

Usamos una malla adecuada en cada caso usando la condición (4.7) y la evolución del tiempo hasta $t=10$. Se obtuvieron los siguientes resultados.

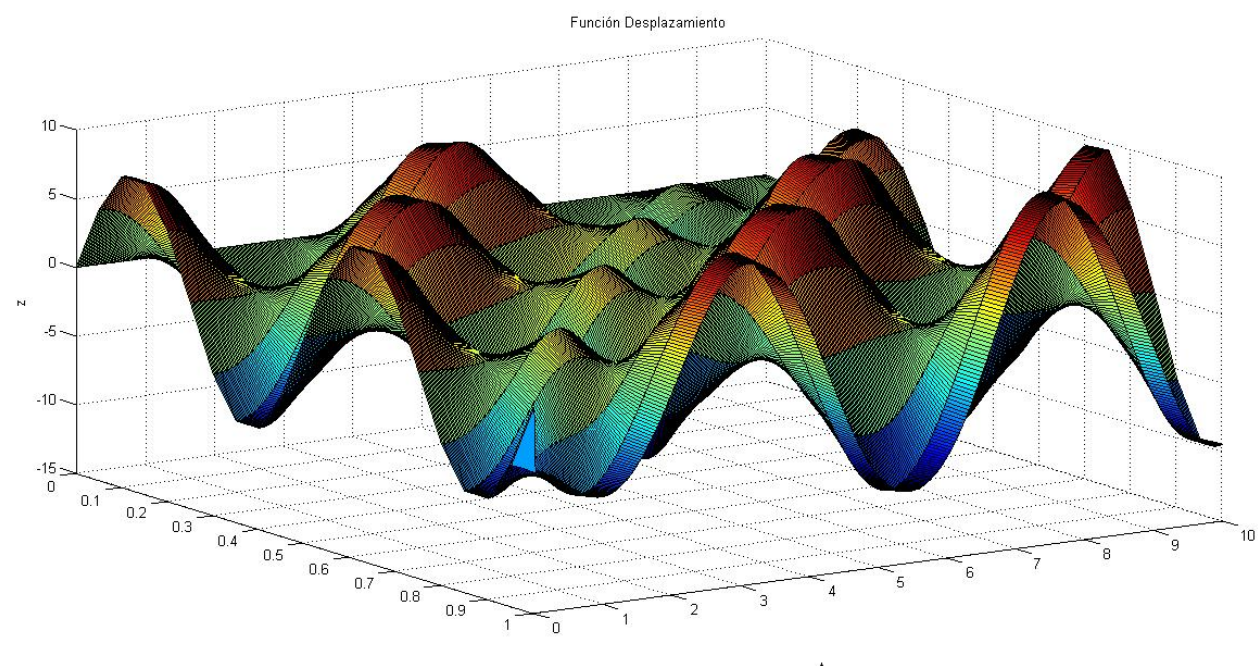

FIGURA 4.1. Función Desplazamiento $\varphi$ para $t=10$.

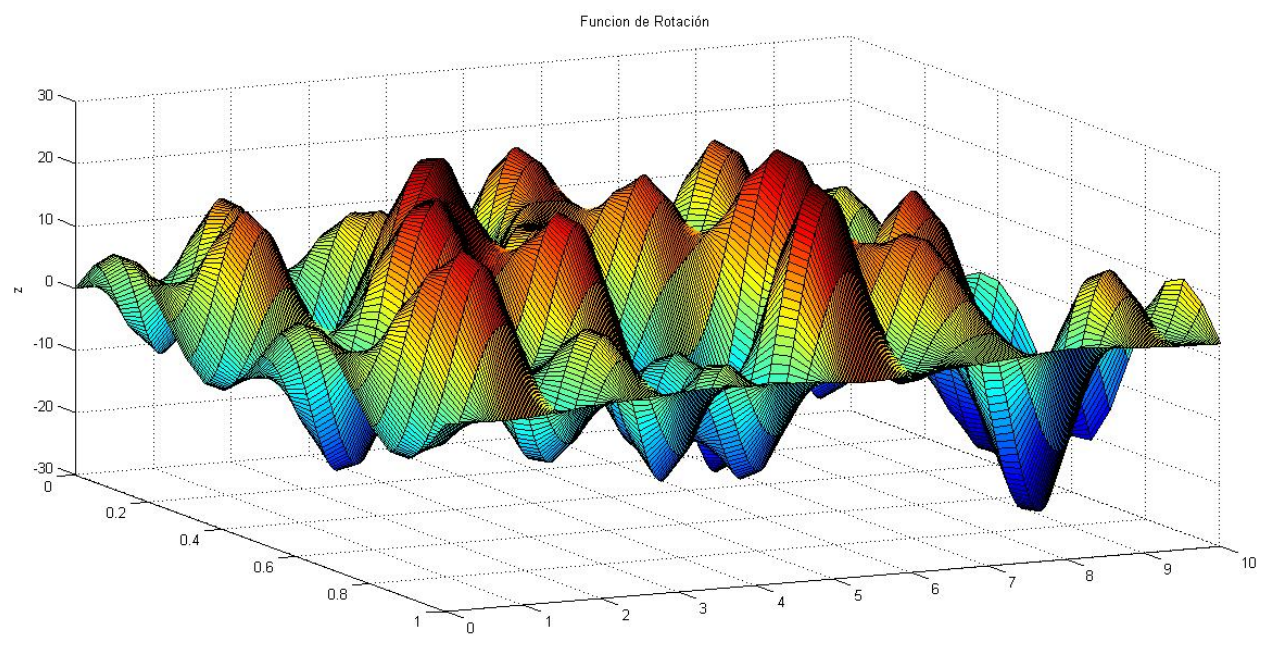

FIGURA 4.2. Función Rotación $\psi$ para $t=10$. 


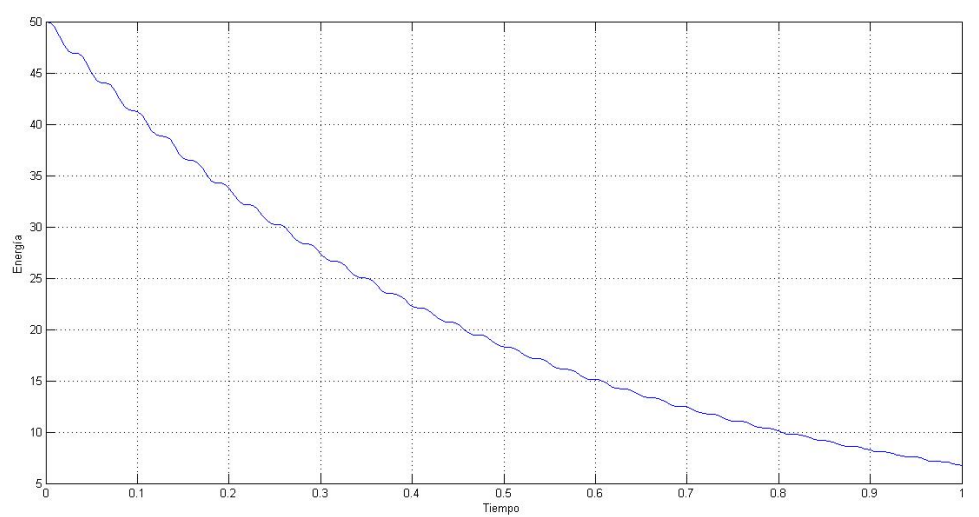

FIGURA 4.3. Decaimiento de la Energía

\section{Conclusiones.}

En este trabajo se hace un análisis donde el resultado principal es la prueba de la estabilidad polinomial del sistema presentado (2.7)-(2.12), usando la teoria de semigrupos y análisis funcional para un mejor enfoque y obtención de estos resultados. Otra herramienta para hacer este análisis es por medio del cálculo variacional. Por lo cual se concluye:

- En lo que respecta a la implementación computacional observamos el comportamiento de las funciones desplazamiento $\varphi$ y de rotación $\psi$, la velocidad de las ondas que suceden en la viga con el pasar del tiempo y la falta de estabilidad exponencial en su decaimiento.

- El decaimiento se da en el momento de flexión ya que el sistema tiene disipación parcial a diferencia de otros sistemas de Timoshenko en donde el decaimiento se da en ambas funciones; debido a esto el decaimiento es lento a diferencia de sistemas con decaimiento exponencial.

- También observamos en la Figura 4.3 la tasa de decaimiento polinomial del problema, con el decaimiento de la energía.

- Para trabajos futuros se implementaría sistemas con memoria y con historia. Se procedería también a hacer un análisis de su comportamiento de estabilidad y su respectiva implementación computacional.

\section{Referencias}

[1] Timoshenko, S., On the correction for shear of the differential equation for transverse vibrations of prismatic bars. Philosophical Magazine. 1921; 41:744-746.

[2] Kim, J.U. and Renardy, Y., Boundary control of the Timoshenko beam. SIAM J. Control Optim. 1987; 25:1417-1429.

[3] Raposo, C.A., Ferreira, J., Santos, M.L., and Castro, N.N.O., Exponential stability for the Timoshenko system with two weak dampings. Appl. Math. Letters. 2005; 18:535-541.

[4] Soufyane, A. and Wehbe, A., Uniform stabilization for the Timoshenko beam by a locally distributed damping. Electron J. Diff. Equations. 2003; 29:1-14.

[5] Kafini, M., General energy decay in a Timoshenko-type system of thermo-elasticity of type III with a viscoelastic damping. J. Math. Anal. Appl. 2011; 375:523-537.

[6] Messaoudi, S.A., and Said-Houari, B. Energy decay in a Timoshenko-type system of thermo-elasticity of type III. J.Math. Anal. Appl. 2008; 348:298-307.

[7] Messaoudi, S.A. and Mustafa, M.I., On the internal and boundary stabilization of Timoshenko beams. NoDEA Nonlinear Differential Equations Appl. 2008; 15:655-671.

[8] Muñoz Rivera, J.E. and Avila, A.I., Rates of decay to non homogeneous timoshenko model with tip body. J. Diff. Equations. $2015 ; 258: 3468-3490$.

[9] Muñoz Rivera, J.E., Estabilização de Semigrupos e Aplicações. LNCC-UFRJ. Brasil. 2009, 1 edição, 120 p.

[10] Acasiete, F. Modelagem Computacional da viga de Timoshenko submetida a cargas pontuais. Dissertação de Mestrado-LNCC. 2016.

[11] Borichev, A. and Tomilov, Y., Optimal polynomial decay of functions and operator semigroups. Math. Ann. 2009; 347:455-478.

[12] Strikwerda, J.C., Finite difference schemes and partial differential equations. SIAM. Philadelphia, 2004, 435 p.

[13] Strauss, W., andA Vasquez, L., Numerical solution of a nonlinear klein-gordon equation. Journal of Computational Physics. $1978 ; 28: 271-278$.

[14] Negreanu, M. and Zuazua, E., Uniform boundary controllability of a discrete 1- $d$ wave equation. System and Control Letters. $2003 ; 48: 261-280$. 